\title{
Charge asymmetry in hadroproduction of heavy quarks
}

\author{
J.H. Kühn and G. Rodrigo \\ Institut für Theoretische Teilchenphysik, Universität Karlsruhe \\ D-76128 Karlsruhe, Germany
}

(February 12, 1998)

\begin{abstract}
A sizeable difference in the differential production cross section of top and antitop quarks, respectively, is predicted for hadronically produced heavy quarks. It is of order $\alpha_{s}$ and arises from the interference between charge odd and even amplitudes respectively. For the TEVATRON it amounts up to $15 \%$ for the differential distribution in suitable chosen kinematical regions. The resulting integrated forward-backward asymmetry of $4-5 \%$ could be measured in the next round of experiments. At the LHC the asymmetry can be studied by selecting appropriately chosen kinematical regions.
\end{abstract}

12.38.Bx, 12.38.Qk, 13.87.Ce, 14.65.Ha

Top quark production at hadron colliders has become one of the central issues of theoretical [1] and experimental [2] research. The investigation and understanding of the production mechanism is crucial for the determination of the top quark couplings, its mass and the search for new physics involving the top system. A lot of effort has been invested in the prediction of the total cross section and, more recently, of inclusive transverse momentum distributions [1].

In this work we will point to a different aspect of the hadronic production process, which can be studied with a fairly modest sample of quarks. Top quarks produced through light quark-antiquark annihilation will exhibit a sizeable charge asymmetry - an excess of top versus antitop quarks in specific kinematic regions - induced through the interference of the final-state with initial-state radiation (Fig. 1 a, b) and the interference of the box with the lowest-order-diagram (Fig. 1 $\mathrm{c}, \mathrm{d}$ ). The asymmetry is thus of order $\alpha_{s}$ relative to the dominant production process. In suitable chosen kinematical regions it reaches up to $15 \%$, the integrated forward-backward asymmetry amounts to $4-5 \%$. Top quarks are tagged through their decay $t \rightarrow b W^{+}$and can thus be distinguished experimentally from antitop quarks through the sign of the lepton in the semileptonic mode and eventually also through the $b$-tag. A sample of hundred to two hundred tagged top quarks should in fact be sufficient for a first indication of the effect.

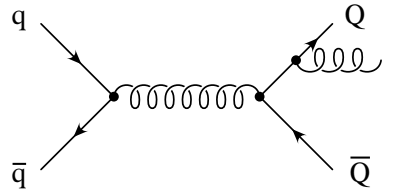

(a)

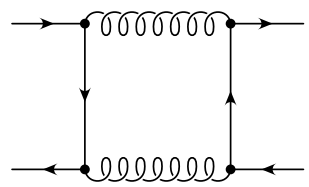

(c)

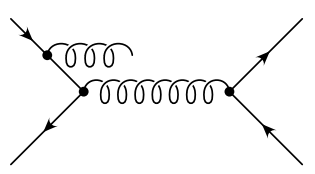

(b)

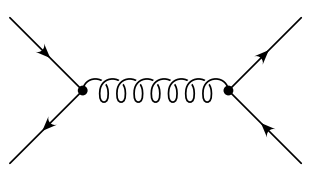

(d)

FIG. 1. Origin of the QCD charge asymmetry in hadroproduction of heavy quarks: interference of final-state (a) with initial-state (b) gluon bremsstrahlung plus interference of the box (c) with the Born diagram (d).

Top production at the TEVATRON is dominated by quark-antiquark annihilation, hence the charge asymmetry will be reflected not only in the partonic rest frame but also in the center of mass system of proton and antiproton. The situation is more intricate for proton-proton collisions at the LHC, where no preferred direction is at hand in the laboratory frame. Nevertheless it is also in this case possible to pick kinematical configurations which allow the study of the charge asymmetry.

The charge asymmetry has also been investigated in [3] for a top mass of $45 \mathrm{GeV}$. There, however, only the contribution from real gluon emission was considered requiring the introduction of a physical cutoff on the gluon energy and rapidity to avoid infrared and collinear singularities. Experimentally, however, only inclusive top-antitop 
production has been studied to date, and the separation of an additional soft gluon will in general be difficult. In this work, we will therefore include virtual corrections and consider inclusive distributions only. We will see below, that the sign of the asymmetry for inclusive production is opposite to the one given for the $t \bar{t} g$ process in [3]. The charge asymmetry of heavy flavour production in quark-antiquark annihilation to bottom quarks was also discussed in [4] 6] where its contribution to the forward-backward asymmetry in proton-antiproton collisions was shown to be very small. In addition there is also a slight difference between the distribution of top and antitop quarks in the reaction $g q \rightarrow t \bar{t} q$. At the TEVATRON its contributions is bellow $10^{-4}$. (This effect should not be confused with the large asymmetry in the top quarks' angular or rapidity distribution in this reaction which is a trivial consequence of the asymmetric partonic initial state and vanishes after summing over the incoming parton beams.)

In a first step the charge asymmetry will be evaluated at the partonic level for the quark-antiquark induced reaction. The calculation proceeds in analogy to the corresponding QED process [7, 8]. The interference terms corresponding to real emission (Fig. 1 $\mathrm{a} * \mathrm{~b}$ ) and virtual radiation (Fig. 1 $\mathrm{c} * \mathrm{~d}$ ) are evaluated separately with an appropriate infrared regulator. Soft radiation up to a cutoff $E_{c u t}^{g}$ is then combined with the virtual correction leaving the hard radiation with $E^{g}>E_{c u t}^{g}$ which can be evaluated numerically. The asymmetric part does not exhibit a light quark mass singularity, whence $m_{q}$ can be set to zero throughout; in other words, no collinear singularities arise. The virtual plus soft radiation on one hand and the real hard radiation on the other contribute with opposite signs, with the former always larger than the later which explains the difference in sign between our result and [3].

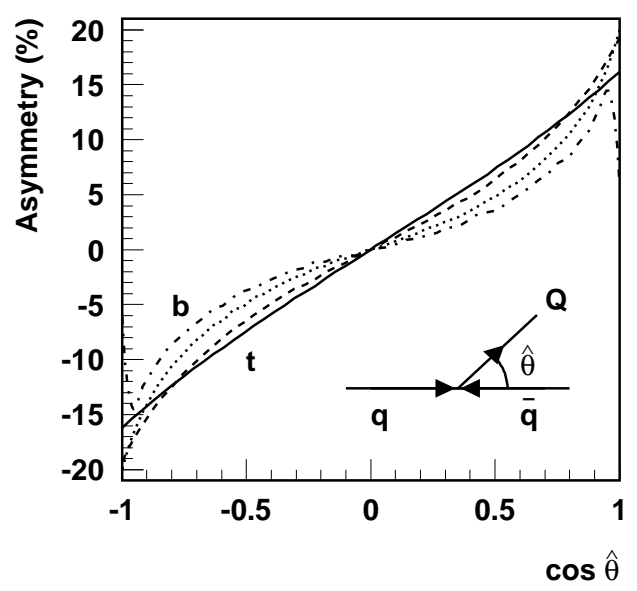

FIG. 2. Differential charge asymmetry in top quark pair production for fixed partonic center of mass energy $\sqrt{\hat{s}}=400 \mathrm{GeV}$ (solid), $600 \mathrm{GeV}$ (dashed) and $1 \mathrm{TeV}$ (dotted). We also plot the differential asymmetry for a b-quark with $\sqrt{\hat{s}}=400 \mathrm{GeV}$ (dashed-dotted).

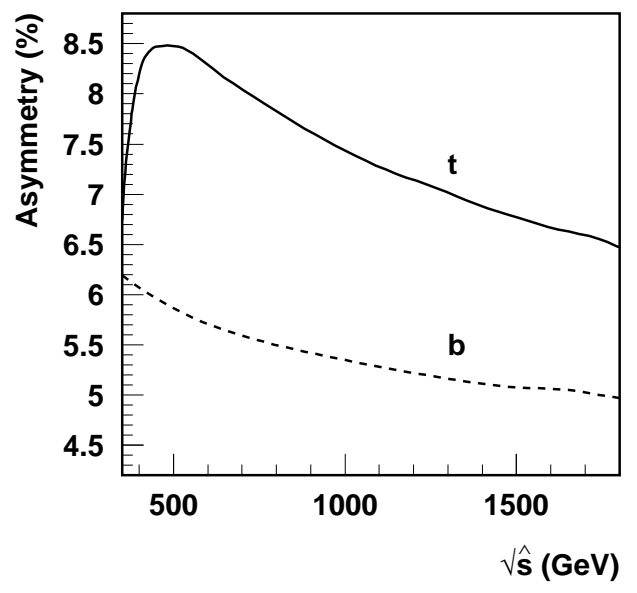

FIG. 3. Integrated charge asymmetry as a function of the partonic center or mass energy for top and bottom quark pair production. 
The QCD asymmetry is related to the corresponding QED asymmetry through the replacement of $\alpha_{Q E D} Q Q^{\prime}$ by the factor $\frac{1}{2} \alpha_{s}\left(d_{a b c} / 4\right)^{2}=\alpha_{s} \cdot 5 / 12$. Let us define the differential asymmetry through

$$
\hat{A}(\cos \hat{\theta})=\frac{N_{t}(\cos \hat{\theta})-N_{\bar{t}}(\cos \hat{\theta})}{N_{t}(\cos \hat{\theta})+N_{\bar{t}}(\cos \hat{\theta})}
$$

where $\hat{\theta}$ denotes the top quark production angle in the $q \bar{q}$ restframe and $N(\cos \hat{\theta})=d \sigma / d \Omega(\cos \hat{\theta})$. Since $N_{\bar{t}}(\cos \hat{\theta})=$ $N_{t}(-\cos \hat{\theta})$ as a consequence of charge conjugation symmetry, $\hat{A}(\cos \hat{\theta})$ can also be interpreted as a forward-backward asymmetry of top quarks. In Fig \&, $\hat{A}(\cos \hat{\theta})$ is displayed for $\sqrt{\hat{s}}=400 \mathrm{GeV}, 600 \mathrm{GeV}$ and $1 \mathrm{TeV}$ for $M_{t}=175 \mathrm{GeV}$. For completeness we also display the result for $b \bar{b}$ production at $\sqrt{\hat{s}}=400 \mathrm{GeV}$ with $M_{b}=4.6 \mathrm{GeV}$. The strong coupling constant is evaluated at the scale $\mu=\sqrt{\hat{s}} / 2$ from $\alpha_{s}\left(M_{Z}\right)=0.118$.

The integrated charge asymmetry

$$
\overline{\hat{A}}=\frac{N_{t}(\cos \hat{\theta} \geq 0)-N_{\bar{t}}(\cos \hat{\theta} \geq 0)}{N_{t}(\cos \hat{\theta} \geq 0)+N_{\bar{t}}(\cos \hat{\theta} \geq 0)}
$$

is shown in Fig 3 as a function of $\sqrt{\hat{s}}$. With a typical value around $6-8.5 \%$ it should be well accessible in the next run of the TEVATRON.

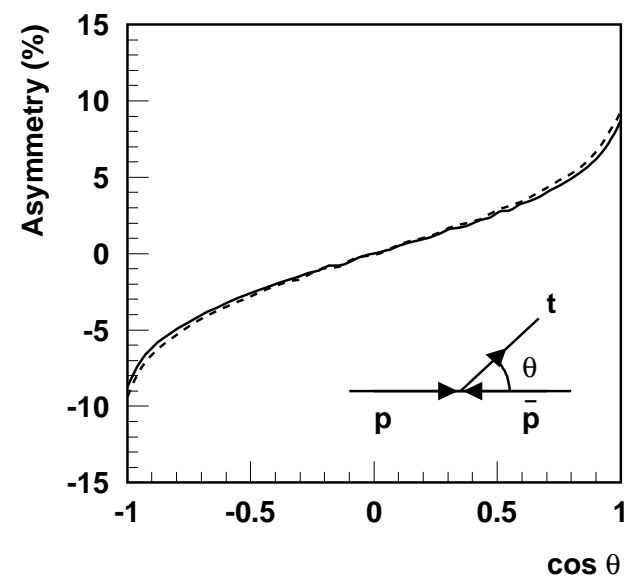

(a)

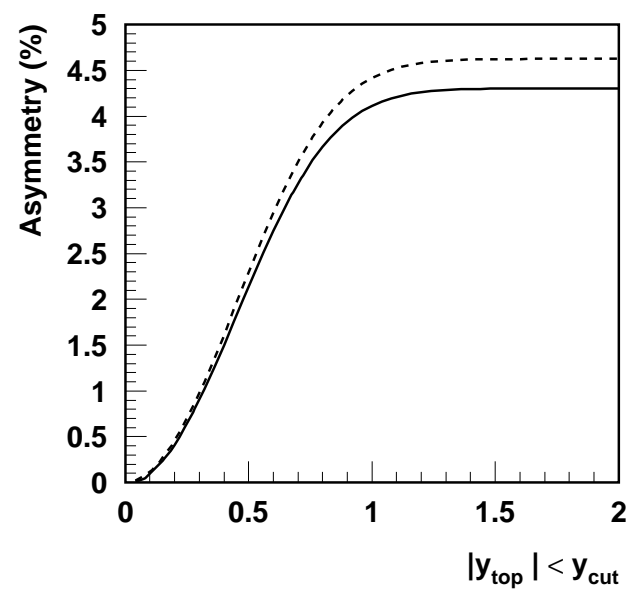

(b)

FIG. 4. a) Differential charge asymmetry in the proton-antiproton restframe using the MRS96-1 structure function. We consider also two different choices of the factorization scale: $\mu=\sqrt{\hat{s}}$ (solid) and $\mu=\sqrt{\hat{s}} / 2$ (dashed). b) Integrated asymmetry for (anti-)top quarks with rapidities less than $y_{\text {cut }}$.

The asymmetry can in principle be studied experimentally in the partonic restframe, as a function of $\hat{s}$, by measuring the invariant mass of the $t \bar{t}$ system plus an eventually radiated gluon. It is, however, also instructive to study the 
asymmetry in the laboratory frame by folding the angular distribution with the structure functions [9]. The differential asymmetry is displayed in Fig. 4 a, where $q \bar{q}$ and $g g$ initiated processes are included in the denominator. For the total charge asymmetry we predict

$$
\bar{A}=\frac{N_{t}(\cos \theta \geq 0)-N_{\bar{t}}(\cos \theta \geq 0)}{N_{t}(\cos \theta \geq 0)+N_{\bar{t}}(\cos \theta \geq 0)}=4.3-4.6 \%,
$$

where different choices of the structure function and different choices of the factorization scale, $\mu=\sqrt{\hat{s}}$ and $\mu=\sqrt{\hat{s}} / 2$, have been considered.

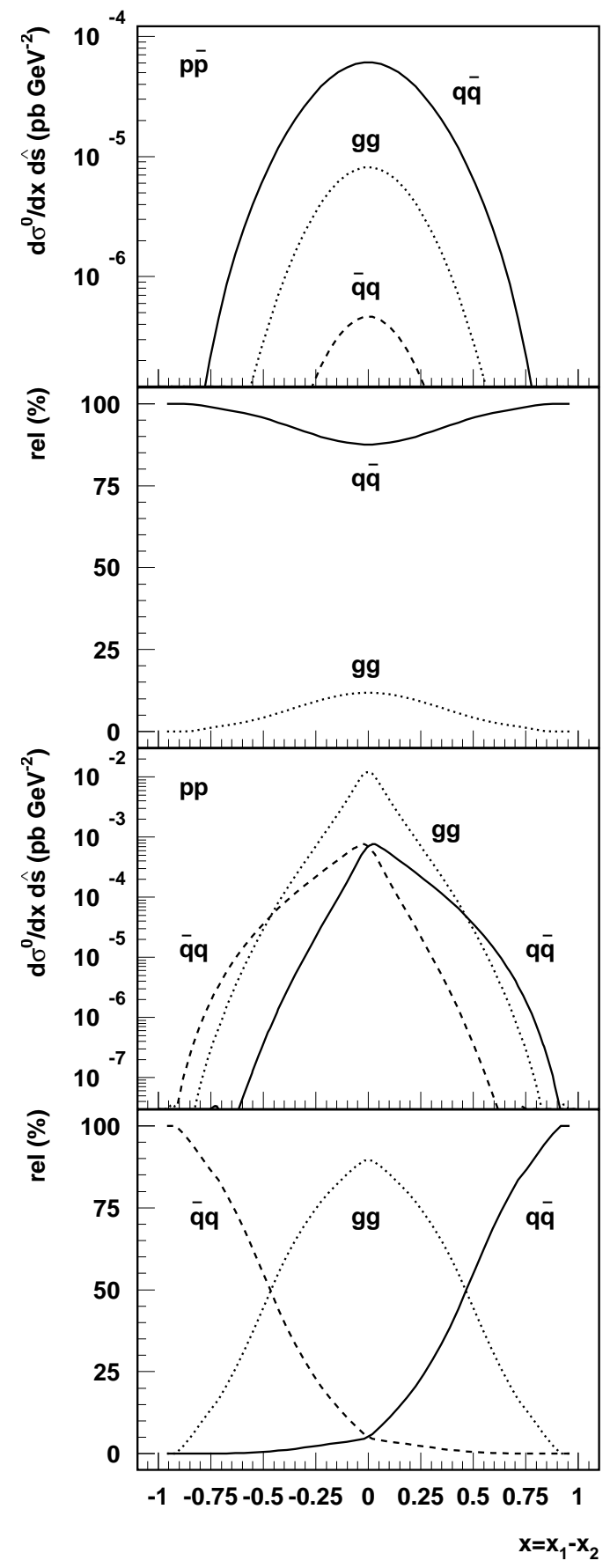

(a)

(b)

(c)

(d)

FIG. 5. Differential cross sections (Fig. a, c) and relative amount (Fig. b, d) of quark-antiquark, antiquark-quark and gluon-gluon initiated processes as functions of $x_{1}-x_{2}=2 P_{3}(t \bar{t} g) / \sqrt{s}$ in lowest order, for $\sqrt{s}=1.8$ TeV in proton-antiproton (Fig. a, b) and $\sqrt{s}=14 \mathrm{TeV}$ in proton-proton (Fig. c, d) collisions with $\sqrt{\hat{s}}=400 \mathrm{GeV}$ in both cases. 
In principle one might expect that cuts on the top quark or its decay products at large rapidities could affect the asymmetry. In Fig $₫ \mathrm{~b}$ we thus present the asymmetry for the restricted range $\left|y_{\text {top }}\right|<y_{\text {cut }}$ as a function of $y_{\text {cut }}$. It approaches its maximal value already for $y_{c u t}=1$, indicating that also cuts on the top decay products $W$ and $b$ jets with rapidities, say, larger than 2 will not lead to a significant reduction of the asymmetry. We would also like to mention that event generators which do not include the full NLO matrix elements [10,11] cannot predict the asymmetry.

Top-antitop production in proton-proton collisions at the LHC is, as a consequence of charge conjugation symmetry, charge symmetric if the laboratory frame is chosen as the reference system. However, by selecting the invariant mass of the $t \bar{t}(+g)$ system and its longitudinal momentum appropriately, one can easily constrain the parton momenta such that a preferred direction is generated for quark-antiquark reactions. This last point is illustrated in Fig 5 where we present the relative amount of quark-antiquark, antiquark-quark and gluon-gluon initiated processes as functions of $x_{1}-x_{2}=2 P_{3}(t \bar{t} g) / \sqrt{s}$ in lowest order, for $\sqrt{s}=14 \mathrm{TeV}$ and $\sqrt{\hat{s}}=400 \mathrm{GeV}$ as characteristical example. A detailed study of this situation will be presented elsewhere [12].

The box diagram, Fig. 1 c, can also give rise to $t t$ in a colour singlet configuration, which in turn interferes with $t \bar{t}$ production through the photon or Z. A similar consideration applies to interference between initial and final state radiation. The resulting asymmetry is obtained from the QCD asymmetry through the following replacement

$$
\frac{\alpha_{s}}{2}\left(\frac{d_{a b c}}{4}\right)^{2} \rightarrow \alpha_{Q E D}\left(Q_{t} Q_{q}+\frac{\left(1-\frac{8}{3} s_{W}^{2}\right)\left(2 I_{q}-4 Q_{q} s_{W}^{2}\right)}{16 s_{W}^{2} c_{W}^{2}} \frac{1}{1-\frac{M_{Z}^{2}}{\hat{s}}}\right)
$$

which amounts to an increase of the asymmetry by typically a factor 1.04 and is thus smaller than uncalculated higher order corrections.

To summarize: the charge asymmetry can be used as an important tool to investigate the production dynamics. For the TEVATRON it amounts to roughly $4-5 \%$ and can therefore be studied with a sample of several hundred $t \bar{t}$ pairs expected for the next run. The asymmetry can also be studied at the LHC if one selects appropriate kinematic configurations.

We would like to acknowledge useful discussions with R.K. Ellis, T. Sjöstrand and M. Seymour. Work supported by BMBF under Contract 057KA92P and DFG under Contract Ku 502/8-1.

[1] S. Catani, "QCD at high-energies," hep-ph/9712442.

[2] P. Tipton, "Experimental top quark physics," Proceedings of the ICHEP 96, Warsaw, Poland, pg.123 (1996).

[3] F. Halzen, P. Hoyer, and C. S. Kim Phys. Lett. 195B (1987) 74.

[4] R. K. Ellis in Strong Interactions and Gauge Theories, ed. J. Tran Thanh Van (Editions Frontière, Gif-sur-Yvette) pg.339 (1986).

[5] P. Nason, S. Dawson, and R. K. Ellis Nucl. Phys. B327 (1989) 49.

[6] W. Beenakker, W. L. van Neerven, R. Meng, G. A. Schuler, and J. Smith Nucl. Phys. B351 (1991) 507.

[7] F. A. Berends, K. J. F. Gaemers, and R. Gastmans Nucl. Phys. B63 (1973) 381.

[8] F. A. Berends, R. Kleiss, S. Jadach, and Z. Was Acta Phys. Polon. B14 (1983) 413.

[9] A. D. Martin, R. G. Roberts, and W. J. Stirling Phys. Lett. B387 (1996) 419, hep-ph/9606345.

[10] G. Marchesini et. al. Comput. Phys. Commun. 67 (1992) 465.

[11] T. Sjöstrand Comput. Phys. Commun. 82 (1994) 74.

[12] J. H. Kühn and R. Rodrigo In preparation. 